\title{
Enfermedad de Devic con respuesta favorable a rituximab: reporte de un caso
}

\author{
Devic disease with favorable response to rituximab: report of a \\ case \\ Orellana Cobos Danilo Fernando1, Berrezueta Córdova Diana Lu- \\ cila', Calle Samaniego Edison Damián'1, Encalada García Carlos \\ Eduardo', Pacurucu Jara Mónica Elizabeth
}

VOLUMEN 37 | No 1 | ABRIL 2019

FECHA DE RECEPCIÓN: 13/01/2019 FECHA DE APROBACIÓN: 17/04/2019 FECHA PUBLICACIÓN: $\quad 30 / 04 / 2019$

1. Hospital Vicente Corral Moscoso

\begin{tabular}{r|l} 
Caso & Clinical \\
Clínico & Case
\end{tabular}

Correspondencia:

dforellanac891ahotmail.com

Dirección:

Juan Jaramillo 4-10

Código Postal:

010104

Teléfono:

0984276134

Cuenca - Ecuador

\section{RESUMEN}

La enfermedad de Devic, también llamada neuromielitis óptica, es una entidad autoinmune desmielinizante del Sistema Nervioso Central, poco común que compromete los nervios ópticos y la médula espinal, ocasionando pérdida de la agudeza visual y afección motora. A continuación, se presenta el caso clínico de una mujer de 25 años, que presenta signos de mielopatía y amaurosis súbitas, cumpliendo criterios para neuromielitis óptica. Se inicia tratamiento con bolos de metilprednisolona sin mejoría, decidiéndose administrar Rituximab con respuesta favorable temprana. EI Rituximab pertenece al grupo de anticuerpos monoclonales anti-CD20, una opción importante ante la falta de respuesta a la primera línea de tratamiento.

Palabras Clave: informes de casos, neuromielitis óptica, rituximab.

\section{ABSTRACT}

Devic's disease, is also called neuromyelitis optica, it is a demyelinating autoimmune entity of the central nervous system, it is uncommon and it compromises the optic nerves and the spinal cord, causing loss of visual acuity and motor impairment. A clinical case of a 25-year-old woman with signs of sudden myelopathy and amaurosis is presented. It is fulfilling criteria for neuromyelitis optica. The treatment with methylprednisolone boluses started without any improvement, and Rituximab was applied with an early favorable response. Rituximab belongs to the group of anti-CD20 monoclonal antibodies, it was an important option in the absence of response to the first line of treatment.

Keywords: case reports, neuromyelitis optica, rituximab 


\section{INTRODUCCIÓN}

La enfermedad de Devic, también conocida como neuromielitis óptica (NMO), es una patología autoinmune desmielinizante del Sistema Nervioso Central, descrita por primera vez en 1894 por Eugene Devic [1]. Aunque fue relacionado como una variante de la esclerosis múltiple, desde 2004 se la diferenció de la misma, asociándose con enfermedades autoinmunes como lupus eritematoso sistémico, síndrome de Sjögren, tiroiditis de Hashimoto, entre otras [2]. Clínicamente, se caracteriza por comprometer a los nervios ópticos y la médula espinal, respetando el cerebro, ocasionando pérdida de la agudeza visual y mielitis transversa [3].

A continuación se presenta un caso clínico de un paciente diagnosticado de enfermedad de Devic, tratado con anticuerpos monoclonales (Rituximab) ante el fracaso terapéuticos con los fármacos de primera línea.

\section{PRESENTACIÓN DEL CASO}

Mujer de 25 años, mestiza, de ocupación comerciante, sin antecedentes patológicos personales ni familiares de relevancia, con un cuadro clínico de seis meses de evolución que inician con dolor lumbar y parestesias en miembros inferiores. Tres meses después manifiesta además parestesias en miembros superiores y hemiparesia izquierda. Dos meses más tarde presenta súbitamente amaurosis izquierda. Cinco días previos al ingreso cursa con paraplejía, retención urinaria, estreñimiento e hipoestesia de T4 a T10. Con una Escala Expandida del Estado de Discapacidad (EDSS) de 18 puntos.

Al examen físico, se observa signos vitales estables: tensión arterial $112 / 60 \mathrm{mmHg}$, frecuencia cardiaca 68 latidos por minuto, frecuencia respiratoria 18 por minuto, temperatura $36.3^{\circ} \mathrm{C}$, saturación $95 \%$ aire ambiente. Funciones mentales superiores conservadas, amaurosis izquierda. Paraplejia y globo vesical. En miembros inferiores: tono muscular disminuido, hiperreflexia, signos de Babinski, Chaddock y Oppenheim presentes. Hipoestesia de T4 a T10, y anestesia en segmentos medulares inferiores. Fondo de ojo izquierdo: papila con palidez marcada, atrofia peripapilar y atrofia óptica.
Exámenes complementarios: biometría y química sanguínea sin alteración; hepatitis $B$, hepatitis $C$, VDRL, VIH, Anticuerpos Antinuceares (ANAs) y Anticoagulante Lúpico negativos; líquido cefalorraquídeo normal.

La tomografía axial computarizada (TAC) de cráneo reportó parénquima sin lesiones, con calcificación fisiológica de plexos coroideos.

La resonancia magnética (RMN) de cráneo y médula espinal mostró discreta hiperintensidad adyacente a las astas posteriores de los ventrículos laterales, visibles en secuencia T2 y FLAIR, nervios ópticos con discreta tortuosidad de predominio izquierdo con leve edema de su vaina (Figura $\mathrm{N}^{\circ} 1$ ). Múltiples regiones hipointensas de segmento largo desde $\mathrm{C} 2$ hasta el borde inferior de T11, compatibles con lesiones de aspecto desmielinizante, que ocupan la mayor parte del diámetro transverso y anteroposterior de la medula espinal, realce de las lesiones de forma heterogénea de predominio en región dorsal tras la administración de contraste, protrusión central del contenido discal de los discos intervertebrales de L4-L5 y L5-S1, sin compromiso radicular (Figura $\mathrm{N}^{\circ} 2$ ).

\section{Figura No 1}

Resonancia magnética de encéfalo

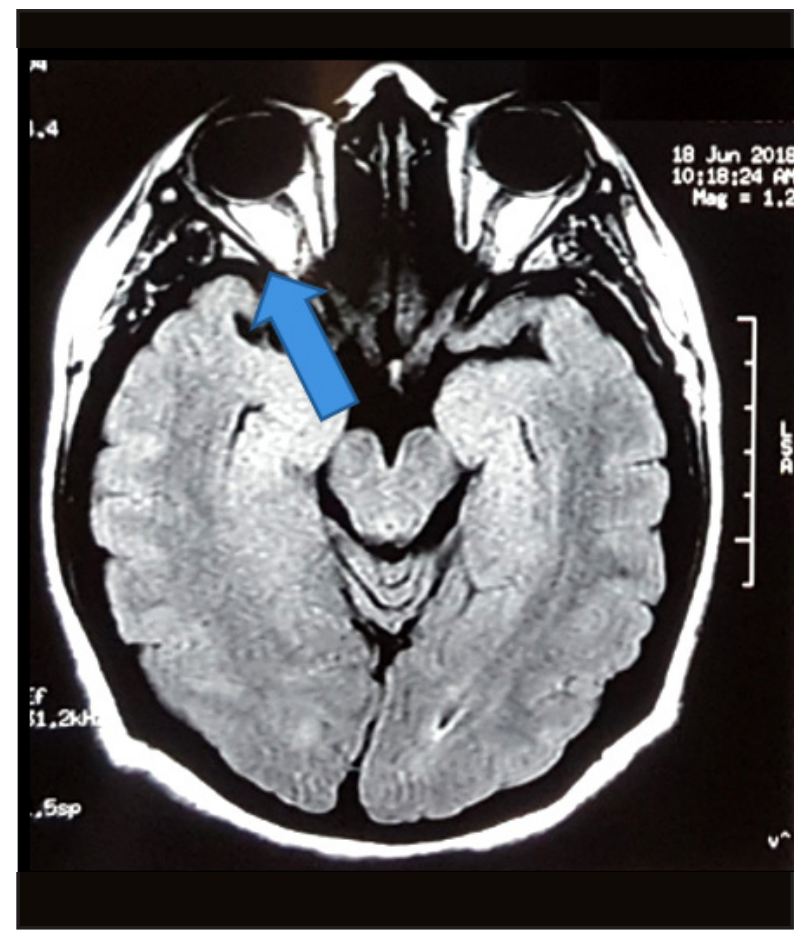




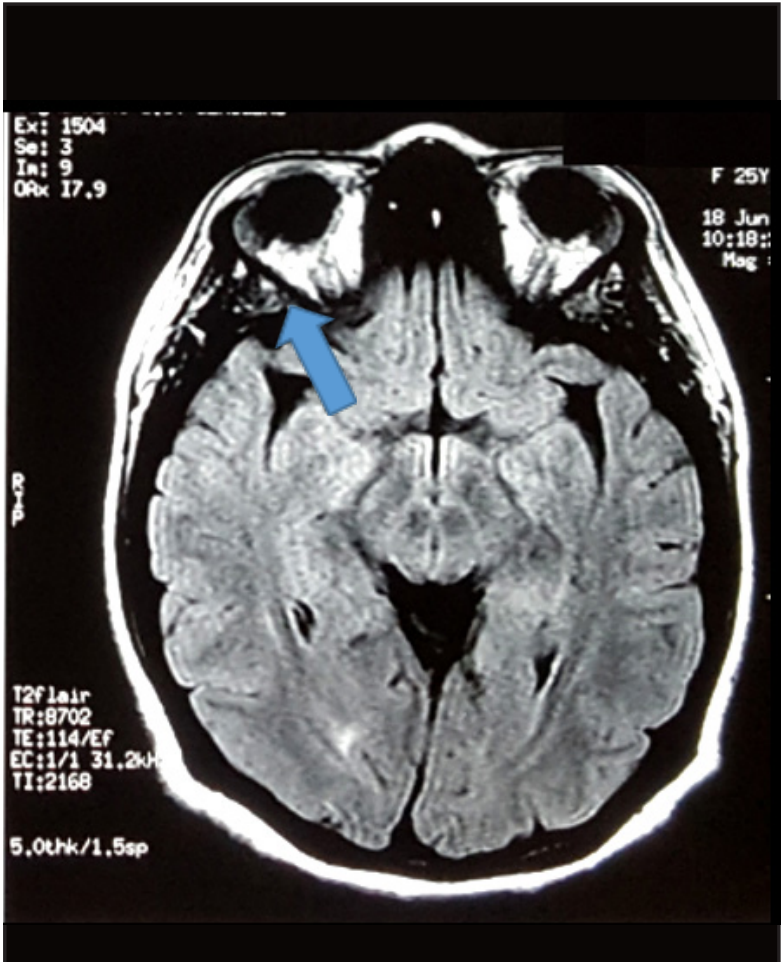

Se aprecia una ligera tortuosidad de los nervios ópticos, de predominio izquierdo.

Figura $\mathrm{N}^{0} 2$

Resonancia magnética simple y contrastada de columna dorsal y cervical.

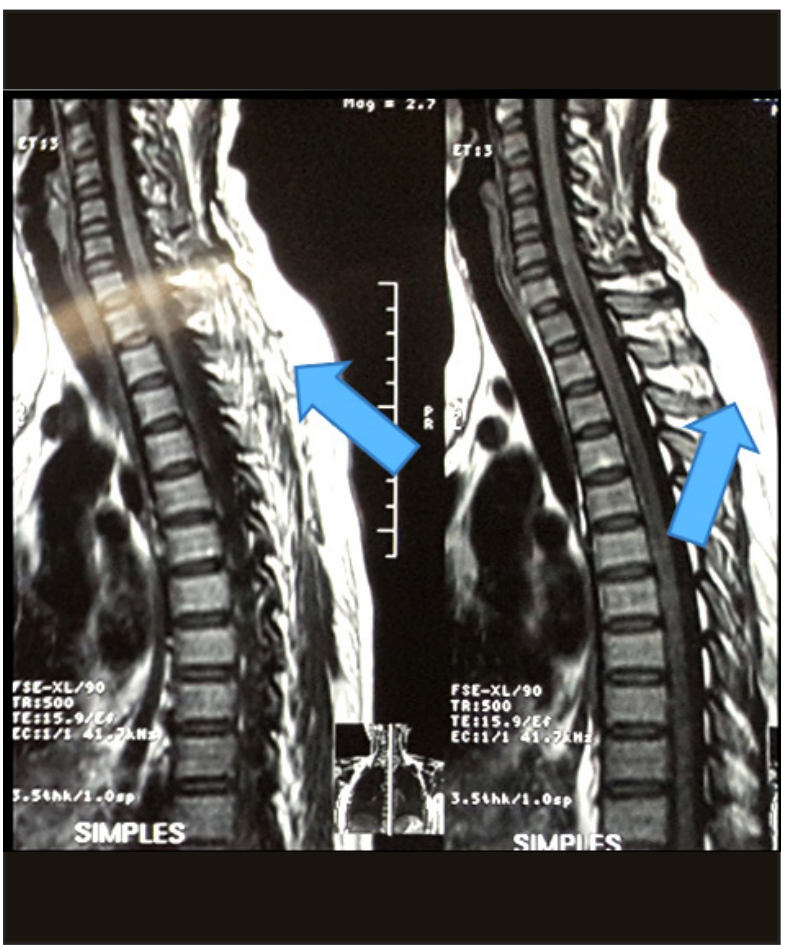

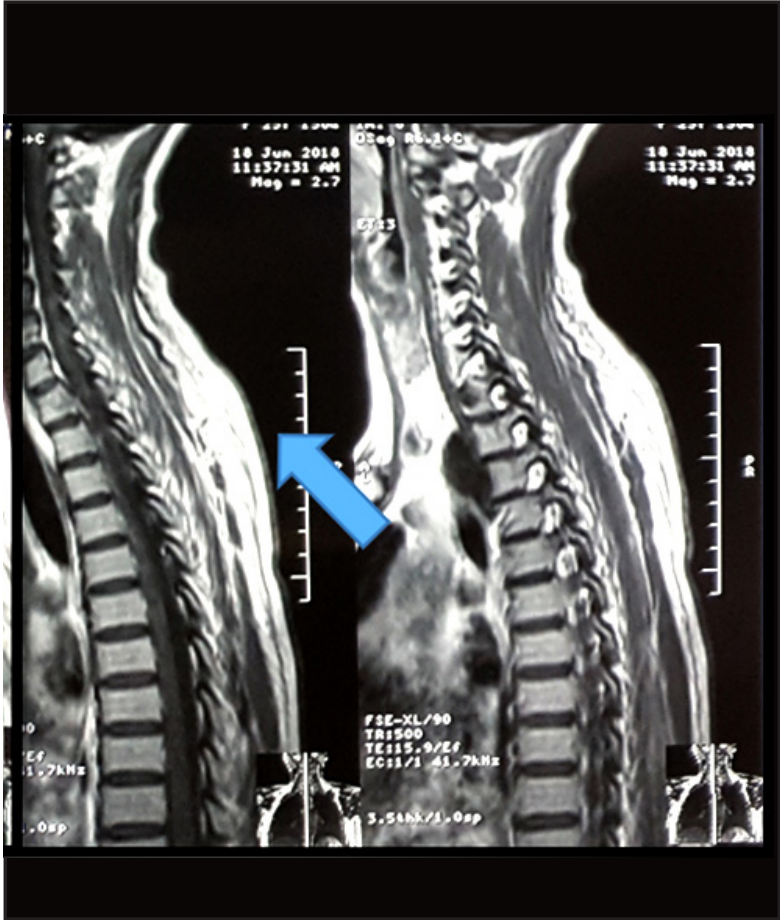

Se observan lesiones de aspecto desmielinizante, intermitentes, desde C2 hasta T11.

Analizada la información presentada se define un cuadro de neuromielitis óptica que inicialmente se medicó con esteroides a base de metilprednisolona, a dosis de 1 gramo intravenoso por cinco ocasiones y posteriormente prednisona oral a $1 \mathrm{mg} /$ $\mathrm{kg} / \mathrm{día}$, sin respuesta clínica. Por lo que luego de una junta médica se decidió la administración de Rituximab (anticuerpo monoclonal anti CD20), $700 \mathrm{mg}$ intravenoso por semana por cuatro semanas.

Al recibir tratamiento con Rituximab, se aprecia una evolución favorable, con mejoría notable de su función motora, recuperación de la fuerza muscular de $4 / 5$ en miembros inferiores que permitió a la paciente la movilización con ayuda de terceros, se produjo un adecuado control de esfínteres, persistió la hipoestesia desde T4 a segmentos medulares inferiores, persiste la amaurosis izquierda.

\section{DISCUSIÓN}

La neuromielitis óptica es una enfermedad inflamatoria desmielinizante autoinmune del sistema nervioso central, caracterizada por afectar principal y selectivamente los nervios ópticos y la médula espinal [4]. 
Hay reportes que estiman una prevalencia de entre 0,3 a 3 casos por cada 100.000 habitantes. En América Latina las cifras no son claras; a nivel mundial se han informado 331 casos, 10 de ellos en Latinoamérica [5]. Es más frecuente en mujeres que en hombres, con una razón de 5:1, con una edad media de comienzo a los 39 años [6].

La fisiopatología de la enfermedad está dada por la producción de anticuerpos tipo IgG por los linfocitos $\mathrm{B}$ y $\mathrm{T}$, cuyo principal blanco inmunitario son los canales de acuaporina 4 (AQP4) de los astrocitos de la medula espinal y nervios ópticos, desencadenando un proceso inflamatorio y desmielinizante en dichas estructuras [7].

En junio de 2015, el Panel Internacional para Diagnóstico de Neuromielitis Óptica estableció los criterios diagnósticos, que se dividen en dos grupos según su estado serológico: aquellos pacientes con anticuerpos anti AQP4 positivos, y aquellos pacientes con anticuerpos anti AQP4 negativos o con estado desconocido [8]. Son seis rasgos clínicos principales los que integran estos criterios: 1) neuritis óptica; 2) mielitis aguda; 3) síndrome del área postrema con hipo y vómitos; 4) síndrome agudo de tronco cerebral; 5) síndrome diencefálico con narcolepsia y lesiones típicas en RMN; 6) síndrome cerebral con lesiones de RMN sugestivas de NMO. Para establecer el diagnóstico debería estar presente uno de los rasgos clínicos señalados y ser positivos los anti-AQP4. Por otra parte, la presencia de dos rasgos clínicos y anti-AQP4 negativos o desconocidos también son criterios diagnósticos; además, sería necesaria la presencia de un curso recidivante y diseminación espacial en la RMN, sin olvidar la exclusión de otros diagnósticos alternativos [9].

Se presenta clínicamente con neuritis óptica y mielitis, con recaída en el $60 \%$ de los casos al año y en el $90 \%$ a los 3 años, generalmente la neuritis óptica precede a la mielitis en meses o años [10].

La neuritis óptica se caracteriza por pérdida de la agudeza visual, dolor a la movilización ocular y discromatopsia en uno o ambos ojos. El campo visual revela escotoma central y el fondo ojo puede ser normal o patológico (edema, atrofia o papila pálida) [11].

La mielitis se presenta con para o tetraplejía, nivel sensitivo, alteración de esfínteres; además de do- lor radicular, espasmos tónicos paroxísticos (recurrentes, dolorosos, con una duración entre 20 - 45 segundos ) y el signo de Lhermitte [12].

El hipo y las náuseas persistentes e intratables se pueden presentar en el $17-43 \%$ de casos, y la afectación respiratoria puede generar paro y muerte por extensión al tronco del encéfalo en un tercio de los pacientes [13].

En cuanto a su tratamiento no hay ensayos clínicos que evalúen la terapéutica, pero según estudios retrospectivos se ha observado buenos resultados con la instauración rápida de corticoides y plasmaféresis. La metilprednisolona juega un papel muy importante siendo el agente de primera línea para suprimir ampliamente la inflamación en recaídas de neuromielitis óptica. La inmunoglobulina intravenosa se ha usado durante mucho tiempo para el tratamiento de una variedad de afecciones neuro-inmunológicas, pero su función en las enfermedades inflamatorias del sistema nervioso central es menos clara $[14,15]$.

En los últimos años el Rituximab, ha sido utilizado en el tratamiento de varias afecciones neurológicas de probable naturaleza autoinmune o en las cuales la inmunidad humoral está implicada. El Rituximab es un anticuerpo que actúa contra las células B CD20+ provocando su depleción de la circulación sanguínea durante 6-8 meses [16].

En el estudio de Kim et al, el protocolo de tratamiento con Rituximab incluyó dos regímenes: 1) $375 \mathrm{mg} / \mathrm{m} 2$ infundido una vez por semana durante 4 semanas; y, 2) 1000 mg infundidos dos veces en un intervalo de 2 semanas, con evolución clínica favorable [17].

Estudios han evidenciado una reducción notable de la discapacidad en estos pacientes con administración de Rituximab, con disminución de recaídas y menor morbimortalidad de la enfermedad $[17,18]$, aunque una desventaja importante es el costo de dicho medicamento [18].

Según estudios, de cada treinta pacientes, el 60\% estaban completamente libres de recaídas durante 5 años al realizar el tratamiento con Rituximab, con dosis semestrales [17]. Se ha realizado estudios con dosis menores de Rituximab (100 mg cada semana durante 3 semanas) debido al coste económico, con resultados satisfactorios [19]. 
Sin embargo, la bibliografía reporta recaídas periódicas caracterizadas por episodios de mielitis con una desmielinización progresiva, por lo que se recomienda continuar con controles, medicación inmunosupresora, terapia física y apoyo psicológico $[19,20]$.

\section{CONCLUSIONES}

La paciente presenta Neuromielitis óptica, con alteraciones visuales y motoras, con una Escala Expandida del Estado de Discapacidad (EDSS) de 18 puntos, tratadas con metilprednisolona intravenosa no presenta una evolución favorable.

Al administrar anticuerpos monoclonales anti-CD20 como Rituximab semanal por cuatro dosis totales, evidencia mejoría notable del cuadro clínico, con una puntuación final en la escala EDSS de 8 puntos.

\section{ASPECTOS BIOÉTICOS}

El presente trabajo se realizó bajo el consentimiento informado de la paciente, con total confidencialidad de datos personales.

\section{INFORMACIÓN DE LOS AUTORES}

- Orellana Cobos Danilo Fernando. Médico General. Posgradista en Medicina Interna. Hospital Vicente Corral Moscoso.

ORCID: http://orcid.org/0000-0001-6269-5512

- Berrezueta Córdova Diana Lucila. Especialista en Medicina Interna. Hospital Vicente Corral Moscoso. ORCID: http://orcid.org/0000-0001-9172-2233

- Calle Samaniego Edison Damián. Especialista en Medicina Interna. Hospital Vicente Corral Moscoso. ORCID: http://orcid.org/0000-0003-3991-9981

- Encalada García Carlos Eduardo. Especialista en Reumatología. Hospital Vicente Corral Moscoso. ORCID: https://orcid.org/0000-0001-5451-5825

- Pacurucu Jara Mónica Elizabeth. Especialista en Neurología. Hospital Vicente Corral Moscoso. ORCID: https://orcid.org/0000-0002-6591-428X

\section{CONTRIBUCIÓN DE LOS AUTORES}

DO, DB, EC, CE: Realizaron la recolección de datos, revisión bibliográfica y redactaron el manuscrito. CE, MP: realizaron el manejo clínico del pacien- te y el análisis crítico del artículo. Todos los autores leyeron y aprobaron la versión final del manuscrito.

\section{CONFLICTO DE INTERESES}

Los autores declaran no tener conflicto de intereses en la presente investigación.

\section{FUENTES DE FINANCIAMIENTO}

Autofinanciado

\section{REFERENCIAS BIBLIOGRÁFICAS}

1. Fabrizzio GC, Gonçalves Júnior E, Cunha KS da, Kahl C, Santos JLG dos, Erdmann $A L$, et al. Care management of a patient with Devic's Disease in Primary Health Care. Rev Esc Enferm USP. 2018;52(1):1-7.

2. Bustillos R, Cortez R, Ramos J, Rodríquez W. Mlelopatía por enfermedad de Devic: Presentación de un caso y revisión de la literatura. Rev Cient Cienc Med. 2015;19(2):4854.

3. Templos LA, Salgado P, Galán N. Manejo del dolor en un caso de neuromielitis óptica (enfermedad de Devic). Rev Soc Esp Dolor. 2016;23(4):186-90.

4. Gutiérrez M, E Bruetman J, Halfon J, Ebner R, Young P. Neuritis óptica bilateral por enfermedad de Devic. Front Med. 2016;11(1):21-4.

5. Bastidas TOZ, Cuellar AV, Quivano JPV, Zuñiga CFV, Hoyos MFV, Paz JCV. Neuromielitis óptica (enfermedad de devic). Medicina (Mex). 2015;37(4):376-84.

6. Uribe-San-Martín R, Ciampi E, Galilea A, Sandoval P, Miranda H, Mellado P, et al. Neuromyelitis optica spectrum disorders: Profile of a cohort according to the 2015 diagnostic criteria. Rev Neurol. 2017;65(5):193-202.

7. Medina Rioja R, Sánchez Jordán A, Bertado Cortes B, Martínez Cortes CE, Martínez Marino M. Devic's disease. A case report and literature review. Rev Fac Med UNAM. 30 de enero de 2018;61(1):26-32. 
8. Meza P C, Henríquez C A, Jara Q A, Canales F P. Aspectos clínicos en el espectro de neuromielitis óptica: revisión de la literatura. Rev Chil Neuro-Psiquiatr. 2016;54(3):22838.

9. Arias M. De la enfermedad de Devic al "espectro de la neuromielitis óptica": una historia inconclusa que se extiende a lo largo de tres siglos. Neurosciences\&History. 2016;4(3):109-16.

10. Larreátegui M, Rodríguez RC, Rosario AD. Neuritis óptica con NMOlgG positiva sin evidencia de mielitis transversa: reporte de caso. Rev Med Pan. 2014;34(2):7-11.

11. Triviño I M, Solanes F, Salgado A C. Neuritis óptica atípica en un adulto joven: un desafío diagnóstico. Rev Chil Neuro-Psiquiatr. 2014;52(2):81-8.

12. Pandit L. Neuromyelitis optica spectrum disorders: An update. Ann Indian Acad Neurol. 2015;18(1):11-5.

13. Kim HJ, Paul F, Lana-Peixoto MA, Tenembaum S, Asgari N, Palace J, et al. MRI characteristics of neuromyelitis optica spectrum disorder. Neurology. 2015;84(11):1165-73.

14. Papadopoulos MC, Bennett JL, Verkman AS. Treatment of neuromyelitis optica: stateof-the-art and emerging therapies. Nat Rev Neurol. 2014;10(9):493-506.

15. Kessler RA, Mealy MA, Levy M. Treatment of Neuromyelitis Optica Spectrum Disorder: Acute, Preventive, and Symptomatic. Curr Treat Options Neurol. 2016;18(1):1-15.

16. Megía MJF, Estruch BC, Miralles FP, Ramos JR, Vicente CA, Andrés JLP. Evaluación del uso de rituximab en la neuromielitis óptica. Neurol Publ Of Soc Esp Neurol. 2015;30(8):461-4.

17. Kim S-H, Jeong IH, Hyun J-W, Joung A, Jo $\mathrm{H}-\mathrm{J}$, Hwang S-H, et al. Treatment Outcomes With Rituximab in 100 Patients With Neuromyelitis Optica: Influence of FCGR3A Polymorphisms on the Therapeutic Response to Rituximab. JAMA Neurol. 2015;72(9):98995.

18. Etemadifar M, Salari M, Mirmosayyeb O, Serati M, Nikkhah R, Askari M, et al. Efficacy and safety of rituximab in neuromyelitis optica: Review of evidence. J Res Med Sci Off J Isfahan Univ Med Sci. 2017;22(18):1-18.

19. Lin J, Li X, Xue B, Tong Q, Chen Z, Zhu W, et al. Low-dosage of rituximab in Chinese patients with neuromyelitis optica spectrum disorder. J Neuroimmunol. 2018;317(1):1-4.

20. Wong E, Vishwanath VA, Kister I. Rituximab in neuromyelitis optica: A review of literature. World J Neurol. 2015;5(1):39-46. 